\title{
Impedance Matching of a Microstrip Antenna
}

\author{
Sameh Khmailia \\ Dept. of Physics \\ El Manar University, Faculty of Sciences \\ Tunis, Tunisia \\ Hichem Taghouti \\ Dept. of Physics \\ El Manar University, Faculty of Sciences \\ Tunis, Tunisia
}

\author{
Sabri Jmal \\ Dept. of Physics \\ El Manar University, Faculty of Sciences \\ Tunis, Tunisia \\ Abdelkader Mami \\ Dept. of Physics \\ El Manar University, Faculty of Sciences \\ Tunis, Tunisia
}

\begin{abstract}
Microstrip patch antennas play a very significant role in communication systems. In recent years, the study to improve their performances has made great progression, and different methods have been proposed to optimize their characteristics such as the gain, the bandwidth, the impedance matching and the resonance frequency.

This paper presents a new method that allows to ameliorate the impedance matching, thus to increase the gain of a rectangular microstrip antenna.

This method is based on the adaptation technique using a simple " $L$ " matching network.

The originality of this work is the exploitation of the principle of causality that permits to detect the problems of reflected waves and to obtain the suitable placement of components that constitute the matching circuit.
\end{abstract}

Keywords-Impedance matching; microstrip antenna; " $L$ " matching network; bond graph model; principle of causality; wave matrix; scattering matrix; transmission and reflection characteristics

\section{INTRODUCTION}

Microstrip patch antennas have been widely researched and developed in recent years. They are used in very specific communication applications such as radars, satellite, broadcasting, radio frequency identification [1].

In its simplest configuration, microstrip antenna consists of a radiating patch on one side of dielectric substrate, which has a ground plane on the other side [2], [3].

Compared with conventional antennas, microstrip patch antennas have better prospects and more advantages. They are lighter in weight, smaller in dimensions and they can be easily integrated with RF and microwave systems [4].

However, microstrip antenna have some drawbacks including narrow bandwidth, low power handling capability, low gain, low impedance matching [5]. But with technology advancements and extensive research into this area, these problems can be gradually overcome.

In this paper, we will present a new impedance matching technique. This method is based on the principle of causality of bond graph and the "L" matching network and permits to improve the impedance matching of a rectangular microstrip antenna.

Firstly, we will present the notion of impedance matching and its different methods.

After that, we will choose the "L" matching network as the best method for improving the impedance matching and the gain of a microstrip antenna.

The principle of causality of bond graph will be exploited to detect the reflected waves and to deduce the appropriate structure of the "L" matching network.

\section{IMPEDANCE MATCHING}

In the high frequency domain, impedance matching is one of the most important applications used to improve the systems performances [6]. It requires adding a matching network between the source and the load of a system in the aim to ensure a maximum power transfer and to avoid the reflected waves [7].

The impedance matching network can be realized using a transformer, a quarter wave line or an "LC" network as " $L$ ", " $T$ " or $\Pi$ form. The most commonly used matching network in RF processing is the " $\mathrm{L}$ " network due to its simplicity, it is constituted only of two components; an inductance and a capacitance that can be controlled for adjusting the real and the imaginary part of the impedance without power loss.

The main principle of any impedance matching scheme is to force the load impedance to look like the complex conjugate of the source impedance, so a maximum power will be transferred to the load.

In RF domain, the first impedance matching concept was related to antenna matching with the aim to obtain desired characteristics such as the gain, the bandwidth and the resonance frequency without modifying the antenna geometry.

In the following, an "L matching network" will be used to improve the impedance matching of a microstrip antenna. 


\section{IMPEDANCE MATCHING OF A MICROSTRIP ANTENNA USING AN "L" NETWORK}

\section{A. Proposed Structure}

The proposed model is given in Fig. 1, it is a microstrip antenna constituted by a substrate characterized by a dielectric constant $\varepsilon_{r}=4.3$ and a thickness $\mathrm{h}=1.575 \mathrm{~mm}$.

A rectangular copper patch characterized by a width $\mathrm{W}=$ $48.5 \mathrm{~mm}$ and a length $\mathrm{L}=37.8 \mathrm{~mm}$ is deposited on one side of the substrate. On the other side is the ground plane.

This antenna is excited through a coaxial cable.

At the resonance frequency, this antenna behaves as a parallel RLC circuit such as given in Fig. 2. The values of RLC elements are determined using the antenna geometric parameters and the substrate characteristics [8].

$$
\begin{aligned}
& S_{e}: \text { Energy source } \\
& R_{G}: \text { The characteristic impedance of generator } \\
& \mathrm{R} 1=20 \mathrm{ohms}, \mathrm{L} 1=27.5 \mathrm{pH} \text { and } \mathrm{C} 1=254.9 \mathrm{pF}
\end{aligned}
$$

The behaviour of an antenna and its performance level can be deduced from the study of its reflection and transmission characteristics as function of frequency [9], [10].

In the following, we will use the "scattering-bond graph method" developed by A. Mami and H. Taghouti to determine the S-matrix describing the relationship between the incident and the reflected waves at the terminals of a system [11], [12].

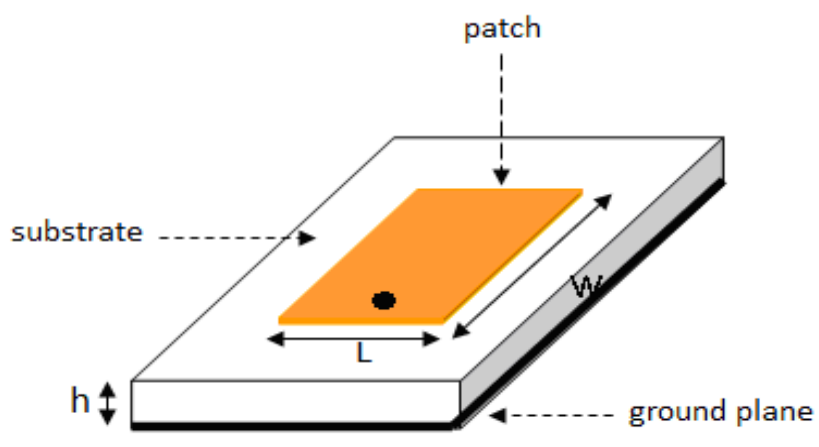

Fig. 1. Microstrip antenna.

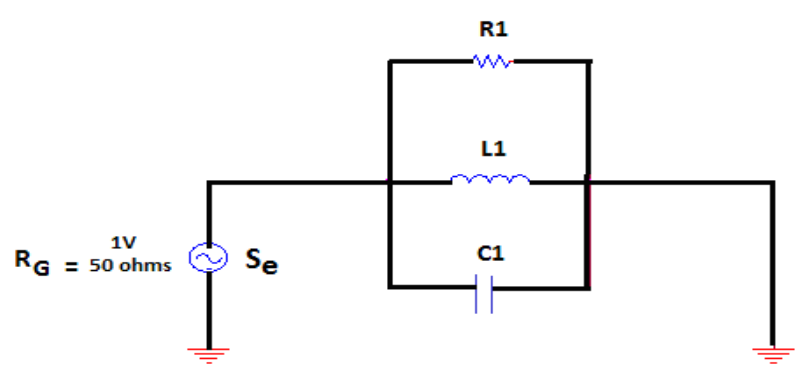

Fig. 2. Electrical model of antenna.

\section{B. Presentation of the Scattering Matrix}

At microwave frequencies, it is difficult to measure the current and the voltage values. Instead, we can measure the transmission and the reflection waves using a network analyzer.

In other words, we can determine the relation between the incident and the reflected waves at each terminal of system to the incident and the reflected waves at all other terminals.

These relationships are completely represented by the scattering matrix called also "S- matrix" [13]-[15].

The problem with S-matrix is that it is not cascadable in its original form, that is to say, one cannot matrix multiply the individual S-matrices of cascaded two port networks to find the total S-matrix.

For this reason a W-matrix is defined. Contrary to $\mathrm{S}$ matrix, this matrix is cascadable, and has a linear transformation relation with the S-matrix.

In the following paragraph, the bond graph model will be exploited to determine the $\mathrm{W}$-matrix, thus the $\mathrm{S}$-matrix of the studied antenna.

\section{Using the Bond Graph to Deduce the S-matrix of the Studied Antenna}

A bond graph is a graphical representation of physical dynamic system [16], [17]. By this approach, a physical system can be represented by symbols and lines identifying the power flow paths.

The lumped elements such as the resistance, the capacitance and the inheritance are interconnected in an energy conserving way by bonds and junctions resulting in a network structure.

From the pictorial representation of the bond graph, the derivation of system equations is so systematic.

The technique that permits to deduce the S-matrix of a system from its bond graph model is called the scatteringbond graph formalism [18].

Taking into account the impedance of generator " $R_{G}$ ", the bond graph model of the studied antenna is given in Fig. 3.

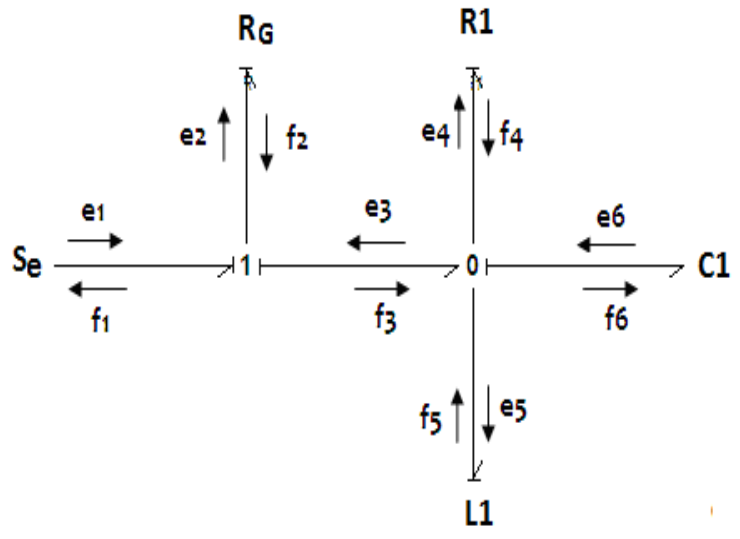

Fig. 3. Bond graph model. 


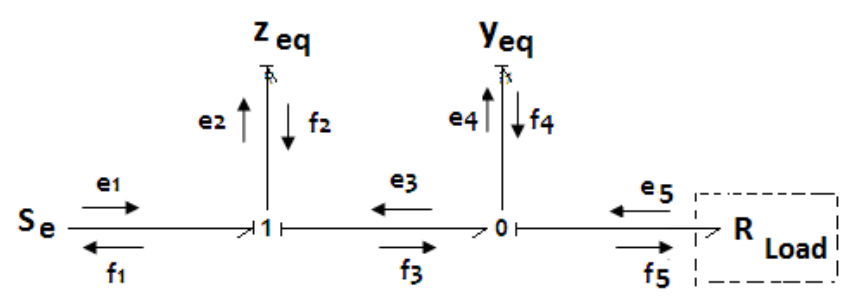

Fig. 4. Reduced bond graph model.

The reduced bond graph model is given in Fig. 4, it is constituted only by an impedance $z_{\text {eq }}$ linked to 1 -junction and an admittance $y_{e q}$ linked to a 0 - junction [19], [20].

$$
R_{\text {Load }} \text { : is an imaginary load }
$$

The resistance of generator is replaced by reduced impedance $z_{\text {eq }}$ linked to a 1 -junction and the parallel elements $\mathrm{R} 1, \mathrm{~L} 1$ and $\mathrm{C} 1$ are replaced by a reduced admittance $y_{e q}$ linked to a 0 -junction.

$$
\begin{gathered}
z_{e q}=r_{g} \\
y_{e q}=\frac{1}{r_{1}}+\frac{1}{\tau_{L 1}}+\tau_{C 1}
\end{gathered}
$$

The normalized values of $R_{G}, \mathrm{R} 1, \mathrm{~L} 1$ and $\mathrm{C} 1$ are given in the following equations:

$$
\begin{gathered}
r_{g}=\frac{R_{G}}{R_{0}} \\
r_{1}=\frac{R 1}{R_{0}} \\
\tau_{C 1}=C 1 . R_{0} \\
\tau_{L 1}=\frac{L 1}{R_{0}}
\end{gathered}
$$

Basing on the bond graph theory, the impedance linked to the 1 -junction can be represented by a wave matrix $W_{1}$ :

$$
W_{1}=\left[\begin{array}{cc}
\frac{z_{e q}+2}{2} & \frac{-z_{e q}}{2} \\
\frac{z_{e q}}{2} & \frac{2-z_{e q}}{2}
\end{array}\right]
$$

The admittance linked to 0 -junction can be represented by a wave matrix $W_{2}$ :

$$
W_{2}=\left[\begin{array}{cc}
\frac{y_{e q}+2}{2} & \frac{y_{e q}}{2} \\
\frac{-y_{e q}}{2} & \frac{2-y_{e q}}{2}
\end{array}\right]
$$

The total wave- matrix $W_{T}$ of the system is obtained by the product of $W_{1}$ and $W_{2}$ :

$$
W_{T}=W_{1} \cdot W_{2}=\left[\begin{array}{ll}
W_{11} & W_{12} \\
W_{21} & W_{22}
\end{array}\right]
$$

To determine the transmission and reflection coefficients, we must determine the Scattering matrix called S- matrix. The following linear transformation that links the $\mathrm{S}$ and $\mathrm{W}$ matrices can be exploited [21], [22], [23].

$$
\left\{\begin{array}{c}
S_{11}=W_{22} W_{12}^{-1} \\
S_{12}=W_{21}-W_{22} W_{12}^{-1} W_{11} \\
S_{21}=W_{12}^{-1} \\
S_{22}=-W_{12}^{-1} W_{11}
\end{array}\right.
$$

Thus, the total S- matrix describing the antenna is given in the following equation:

$$
S=\left[\begin{array}{ll}
S_{11} & S_{12} \\
S_{21} & S_{22}
\end{array}\right]=\left[\begin{array}{cc}
W_{22} W_{12}^{-1} & W_{21}-W_{22} W_{12}^{-1} W_{11} \\
W_{12}^{-1} & -W_{12}^{-1} W_{11}
\end{array}\right]
$$

The adaptation level of the antenna is determined from the characteristic of the reflection coefficient S11 as function of frequency given in Fig. 5.

According to the variation characteristic of S11 as function of frequency, the impedance matching is not ensured. In fact, in the antenna theory, a microstrip antenna is considered adapted only if the absolute value of S11 is greater than or equal to $15 \mathrm{~dB}$.

To remedy this problem, thus to ensure a maximum power transfer, we will use the "L" matching network. The technique is explained in the following paragraph.

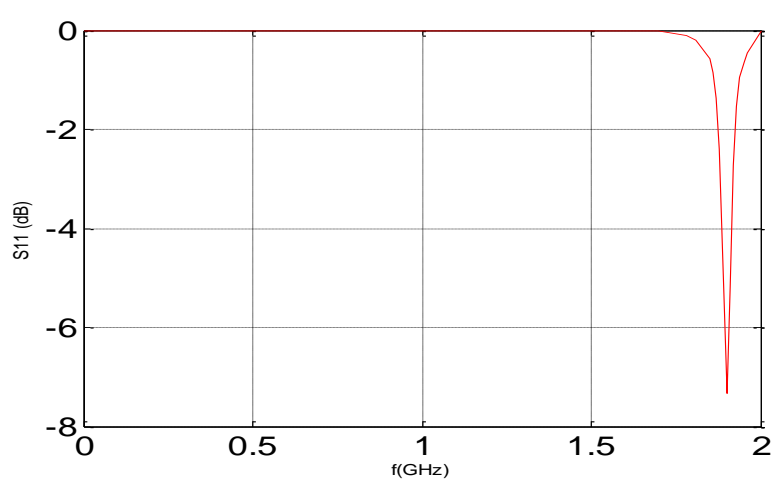

Fig. 5. Reflection coefficient S11 as function of frequency. 


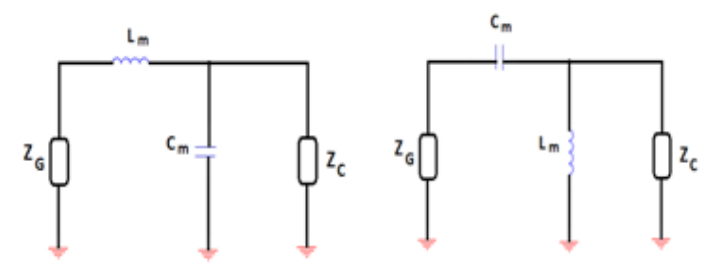

(a)

(b)

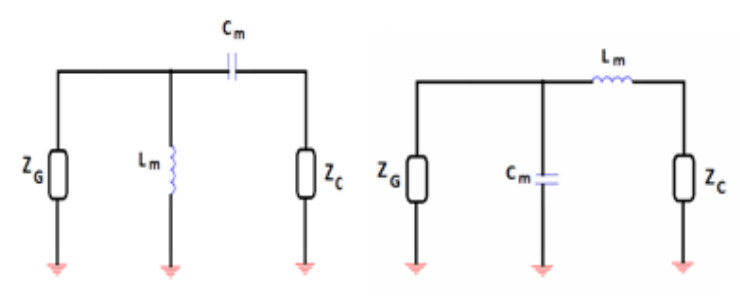

(c)

(d)

Fig. 6. (a, b, c, d): Four configurations of L matching network.

\section{D. "L" Matching Network Application}

The "L" match circuit gets its name because the circuit topology can look like the letter "L". It is one of the easiest lossless ways of matching the source impedance to the load impedance.

The four configurations containing "L" matching network given in Fig. 6 are possible.

$Z_{G}:$ is the impedance of the generator

$Z_{C}$ : represents the characteristic impedance of antenna

$L_{m}:$ Inductance of "L" matching network

$C_{m}:$ Capacitance of "L" matching network

The concept of causality of bond graph can be used to choice the more appropriate disposition of the capacitance $C_{m}$ and the inductance $L_{m}$ in the L matching network [24]. In fact, the principle of causality imposes that:

- For 0-junctions, one of the bonds sets the effort for the rest, so only one causal stoke is on the junction, while the others are away from it.

- For 1-junctions, one of the bonds sets the flow for the rest, and its effort is computed from them, so all but one of the causal stokes are on the junction, while the remaining one is away from it.

Using the bond graph model, the previous four representations of matched antenna can be represented such as given in Fig. 7(a, b, c, d).

According to the principle of causality, for 0 -junction, only one causal stroke must be on junction, while others must be away from it. This principle is not respected in the models given in Fig. 7(a) and 7(b).
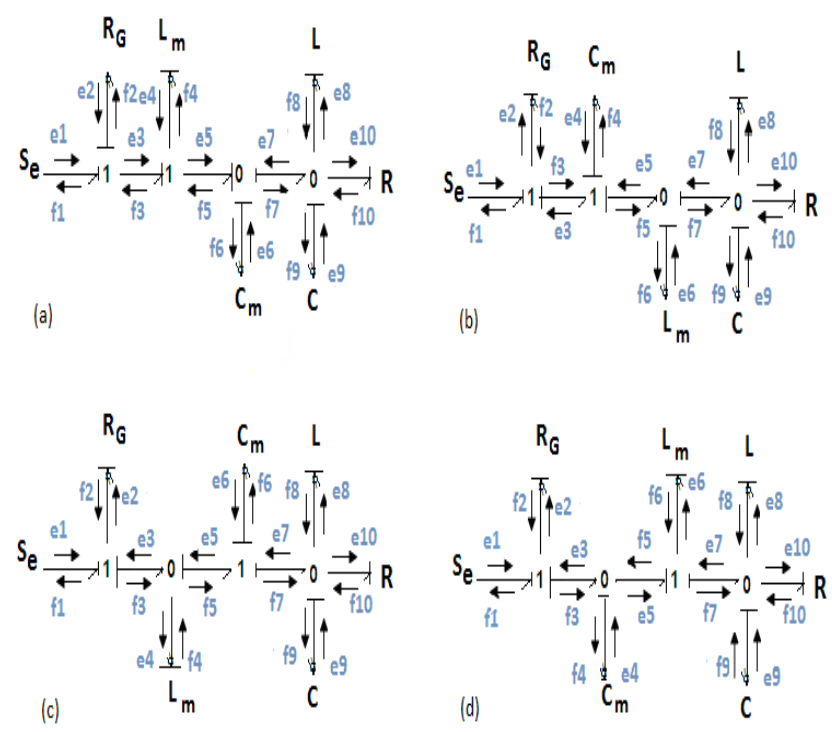

Fig. 7. (a, b, c, d): Bond graph models of four matched antenna configurations.

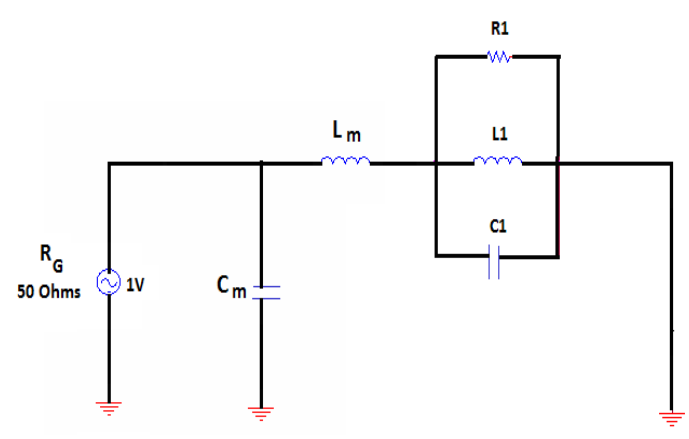

Fig. 8. Electric model of matched antenna.

Thus, we will choose between the structure given in Fig. 7(c) and 7(d).

If we choose the model given by Fig. 7(d), the electric model of matched antenna is given in Fig. 8.

To find the values of $\mathrm{L}_{\mathrm{m}}$ and $\mathrm{C}_{\mathrm{m}}$ at a resonance frequency $f_{r}=1.9 \mathrm{GHz}$, we use the equations given below [25]:

$$
\begin{gathered}
C_{m}=\frac{1}{2 \pi f_{r}} \sqrt{\frac{R_{1}-R_{2}}{R_{2}}} \\
L_{m}=\frac{R_{2}}{2 \pi f_{r}} \sqrt{\frac{R_{1}-R_{2}}{R_{2}}}
\end{gathered}
$$

Over a frequency range from 0 to $2 \mathrm{GHz}$, we have compared the results of the variation of the reflection coefficient S11 as function of frequency with and without the "L" matching network. The result is shown in Fig. 9. 


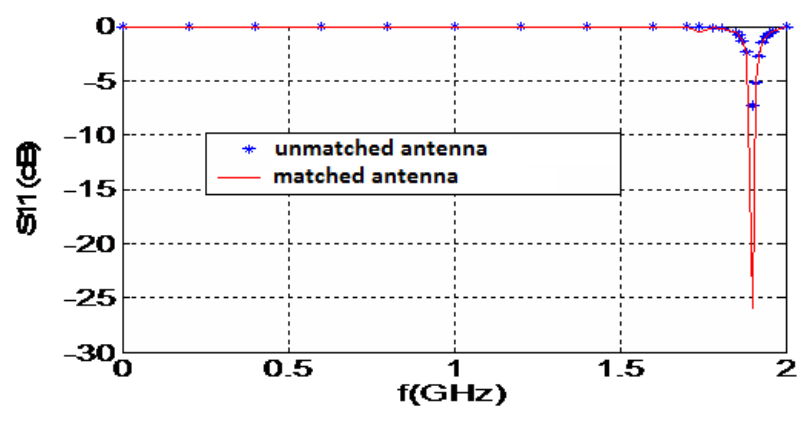

Fig. 9. S11 as function of frequency.

At the resonant frequency, the absolute value of S11 has gone up from 7.5 to $26 \mathrm{~dB}$.

The "L" matching network permitted to improve considerably the gain of the microstrip antenna which is explained by a good impedance matching.

\section{CONCLUSION}

Impedance matching is a widely used application in high frequency circuit design. It is concerned with matching one part of a circuit to another in order to achieve a maximum power transfer between the two parts and to minimize the reflected waves. There are different impedance matching network such as the "L" matching network, the transmission line matching circuit and the quarter wave impedance transformer.

In this work, we used the " $\mathrm{L}$ " matching network to match the impedance of a microstrip antenna, thus to improve its gain and resonant frequency. This choice is due to the simplicity, low cost and ease of design of an "L matching network"; it is only constituted by an inductance and a capacitance.

The principle of causality is used to detect the problem of reflected waves and to choice the more appropriate disposition of the "L" circuit components.

The method presented in the work of A. Mami and H. Taghouti is exploited to determine the scattering matrix of the antenna.

The simulation result of the reflection coefficient S11 as function of frequency with and without impedance matching shows that the gain of adapted antenna is more important.

\section{REFERENCES}

[1] K. Rama Devi, A. Mallikarjuna Parasad and A. Jhansi Rani, "Design of Pentagon Microstrip Antenna for Radar Alimenter Application," International Journal of Web \& Semantic Technology, IJWest, Vol.3, No.4, October 2012.

[2] Anushi Arora, Aditya Khemchan dani, Yash Rawat, Shashank Singhai, Gaurav Chaitanya," "Comparative Study of different Feedin Techniques for Rectangular Microstrip Patch Antenna," International Journal of innovative Research in Electrical, Electronics, Instrumentationand control Engineering Vol 3, Issue 5, May 2015.

[3] Amri Sigh, " Design of square patch microstrip antenna for circular polarization usgin IE3D Software", National Institute of thechnology Rourkela Orissa India.
[4] S.M. Yang, C.H. Huang, C.C. Hong, "Design and analysis of microstrip antena arrays in composite Laminated Substrates" Journal of Electromagnetic Analysis and Applications, pp. 115-124, 2014.

[5] J. Ankur Kaushal, Sachin Tyagi," Microstrip Patch antenna its Tyoe, merits, demerits and its applications "International Jounal of Engneering Sciences \& Research Technology, July 2015.

[6] Thesis of Vishwanath Iyer, "Broad band impedance matchinng of antenna radiators," The faculty of Worcester polytechnic institute in Electrical and Computer Engineering .

[7] Thesis of Victor Freitas, "Etude et réalisation de réseaux d'adaptation d'impédance accordables linéaires et non linéaires sur PCB et silicium CMOS pour des applications en radiofréquences," Grenoble University, 2006.

[8] Wolff, I and Knoppik, N, "Rectangular and circular microstrip disk capacitors and resonators," IEEE Trans, 1974, MTT-22, pp, 857- 864

[9] A.M.Niknejad" Two-port networks and amplifiers", EECS 142.

[10] F.Caspers"RF engineering basic concepts: S- parameters", CERN, Geneva Switzerland .

[11] H.Taghouti, and A.Mami, "Discussion around the Scattering Matrix Realization of a Microwave Filter using the Bond Graph Approach and Scattering Formalism". American Journal of Applied Sciences, Vol. 9, Issue. 4, 2012.

[12] H.Taghouti, and A. Mami, "Modeling Method of a Low-Pass Filter Based on Microstrip T-Lines with Cut-Off Frequency $10 \mathrm{GHz}$ by the Extraction of its Wave-Scattering Parameters from its Causal Bond Graph Model”. Am. J. Eng. Applied Sci., Vol. 3, pp. 631-642, 2010.

[13] Laurent Chusseau, "Paramètres S- Antennes", Centre d'électronique et de Microélectronique de Monpellier.

[14] TAGHOUTI, H. et MAMI, A, "Extraction, Modelling and Simulation of the Scattering Matrix of a Chebychev Low- Pass Filter with cut-off frequency $100 \mathrm{MHz}$ from its Causa and Decomposed Bond Graph Model", ICGST International Journal on Automatic Control and System Engineering. Vol. 10, Issue I, pp. 29-37, 2010.

[15] JANUSZ A. DOBROWOLSKI, "Microwave Network Design Using the Scattering Matrix", Artech House.

[16] Masson, S.J," Feedback theory: Further properties of signal flow graphs", Monographs in modern engineering science New York, Mc Graw Hill, 1964, pp.920-926.

[17] BREEDVELD, P.C, "Computer-aids for Modelling and Conceptual Design', IEEE SMC'93, Int. Conf. on Systems Man and Cybernetics. Le Touquet, Vol. 2, pp. 209-215, Oct. 1993.

[18] Amara, M and Scavarda, S, " A procedure to match bond graph and the scattering formalism", J. of the Franklin Inst, Vol.328, $N^{\circ} 516$, pp. 887899, 1991.

[19] S. Khmailia, H. Taghouti, R. Mehouachi and A. Mami, "Application of the reduced bond graph approach to determine the scattering parameters of a matching network of a planar inverted $f$ antenna", International Journal of Advances in Engineering \& Technology, Vol. 3, Issue 2, pp. 77-89. May 2012.

[20] C.Sueur and G.Dauphin- Tanguy, "Bond-graph approach for structural analysis of mimo linear systems", Journal of the Franklin institute, Volume 328, Issue 1, 1991, Pages 55-70.

[21] Sameh KHMAILIA, Hichem TAGHOUTI \& Abdelkader Mami," AStudy of A Band Stop Fillter", European Journal Of Scientific Research, Vol. 95 No 4 pp.582-588, February 2013.

[22] Riadh MEHOUACHI, Hichem TAGHOUTI, Sameh KHMAILIA,\& Abdelkader Mami,"Modeling and Simulation of the Patch Antenna by Using a Bond Graph Approach", International Journal of Advances in Engineering \& Technology, Vol. 2, Issue 1, pp.474-484, Jan 2012.

[23] Raul G. Longoria, "Wave-scattering formalisms for multiport energetic systems", J.Franklin Inst.vol.333(B).N4,pp.539-564, 1996.

[24] Rosenberg, R.c "Exploding Bond Graph Causality in physical Systems Models" Trans. ASMEJ. OF Dyn. Sys. Meas. And Control" 1987 vol.109, N 4, pp.206- 2012.

[25] François de Dieuleveut, Olivier Romain "Eléctronique appliquée aux hautes fréquences". 